\title{
INTERSECTIONS OF MINIMAL SUBMANIFOLDS IN MANIFOLDS OF PARTIALLY POSITIVE CURVATURE
}

\author{
Katsuei Kenmotsu and Changyu Xia*
}

\section{Introduction and main theorems}

In 1961, T. Frankel [3] showed that two compact totally geodesic submanifolds $P$ and $Q$ in a Riemannian manifold $N$ of positive sectional curvature must necessarily intersect if their dimension sum is at least that of $N$. If $N$ is also a Kaehler manifold, he proved a more satisfactory result: There, instead of requiring $P$ and $Q$ to be totally geodesic, under the assumption that they are analytic submanifolds, he obtained the same conclusions under the assumptions. The proof was done by a nice application of the formula for the second variation of arc-length of a geodesic derived by Synge in [11].

Synge once used the second variational formula to prove that an even dimensional orientable compact manifold with positive sectional curvature is simply connected. It turns out that the Synge's method for the second variation of arc-length of a geodesic works as a powerful tool in differential geometry. In the present paper, we shall study the intersections of minimal submanifolds in a Riemannian manifold with partially positive curvature and analytic submanifolds in a Kaehler manifold with partially positive holomorphic bisectional curvature by using this method. We remark that Galloway and Rodriguez [7] studied the same problems for minimal submanifolds in a manifold with non-negative sectional curvature by a different approach but our results have few relations with theirs.

Before stating our theorems, we recall some concepts of partially positive curvature (cf. [12]). Let $N^{n}$ be an $n$-dimensional Riemannian manifold and $p \in N^{n}$ be a point of $N^{n}$. If, for any $(k+1)$ mutually orthogonal unit tangent vectors $e, e_{1}, \cdots, e_{k} \in T_{p} N^{n}$, we have $\sum_{l=1}^{k} K\left(e \wedge e_{\imath}\right)>0$ (resp. $\geqq 0$ ), we say $N^{n}$ has positive (resp. nonnegative) $k$-th Ricci curvature at $p$. If $N^{n}$ has positive (resp. nonnegative) $k$-th Ricci curvature at every point of it, we call $N^{n}$ has positive (resp. nonnegative) $k$-th Ricci curvature and denote this fact by $\operatorname{Ric}_{(k)}\left(N^{n}\right)>0$ (resp. $\left.\geqq 0\right)$. Here, $K\left(e \wedge e_{\imath}\right)$ denotes the sectional curvature of the

1991 Mathematics Subject Classification: 53C42, 53C40

* Supported by the JSPS postdoctoral fellowship.

Received February 10, 1994. 
plane spanned by $e$ and $e_{\imath}(1 \leqq i \leqq k)$. Thus, positive (nonnegative) 1 -th Ricci curvature is equivalent to positive (nonnegative) sectional curvature and positive (nonnegative) (n-1)-th Ricci curvature is equivalent to positive (nonnegative) Ricci curvature. Compact locally symmetric spaces of rank $\geqq 2$ are examples of manifolds with positive $k(k>1)$-th Ricci curvature. Slight perturbations of these metrics give non-symmetric examples. Further examples of manifolds of positive $k$-th Ricci curvature can be found among the compact homogeneous spaces with a bi-invariant metric (cf. [1]).

Now we can state our Theorem 1 as follows:

THEOREM 1. Let $N$ be an n-dimensional complete connected Riemannian manifold with nonnegative $k$-th Riccl curvature. Let $V$ and $W$ be a complete immersed totally geodesic hypersurface and an $r(\geqq k)$-dimensional complete $\imath m$ mersed minimal submanifold of $N$, respectively, each immersed as a closed subset, and let one of $V$ and $W$ be compact. Assume $N$ has positive $k$-th Ricci curvature either at all points of $V$ or at all points of $W$. Then $V$ and $W$ must intersect.

Theorem 1 generalizes several known results. Firstly, it generalizes a Myers' theorem [10] stating that there exists no closed immersed minimal submanifolds in an open hemisphere, since as an immediate consequence of Theorem 1 we know that every closed immersed minimal submanifold in a Riemannian manifold $N$ of positive sectional curvature must have non-empty intersection with any immersed closed totally geodeisc hypersurface of $N$. Secondly, Theorem 1 is a higher dimensional generalization of the following Hadamard's theorem [5]: On a complete connected surface with positive curvature, every geodesic must meet every closed geodesic on the surface. Finally, it also generalizes the Frankel's theorem mentioned before in case of one of the submanifolds $P$ and $Q$ has codimension one. We would like to mention that the Frankel's theorem can be also regarded as a generalization of the above Hadamard's theorem.

Theorem 1 is optimal in the sense that the condition on $V$ can't be weakened either to "minimal hypersurface" or to "totally geodesic submanifold" (see Example 1) and that one can't expect the same conclusion in Theorem 1 still holds if $N$ is only assumed to have nonnegative $k$-th Ricci curvature (see Example 2).

As an application of Theorem 1, we can prove certain nonexistence result for minimal immersions into a half space of a Riemannian manifold $N$ with partially positive curvature. Namely, we have

THEOREM 2. Let $M^{n-1}$ be an orientable embedded compact totally geodesic hypersurface in an n-dimensional orientable complete connected Riemannian manifold $N^{n}$ with nonnegative sectional curvature. Assume $N^{n}$ has positive $k$-th Ricci curvature at all points of $M^{n-1}$. If $V^{r}$ is an immersed closed $r(\geqq k)$-dimensional minimal submanifold of $N^{n}$ which is on one side of $M^{n-1}$, then $V^{r}$ is contained 
in $M^{n-1}$. Moreover, when $r=n-2$, the natural homomorphism of the fundamental groups: $\pi_{1}\left(V^{n-2}\right) \rightarrow \pi_{1}\left(M^{n-1}\right)$ is surjective.

The simplest illustration of Theorem 2 is perhaps the following: Let $S^{2 m+1}(1)$ be the standard unit $(2 m+1)$-sphere and $R P^{2 m+1}(1)$ the $(2 m+1)$-dimensional real projective space of curvature 1 . Here, $m \geqq 1$. Let $N=S^{2 m+1}(1) \times$ $R P^{2 m+1}(1)$ be the Riemannian product space of $S^{2 m+1}(1)$ and $R P^{2 m+1}(1)$. It is easy to check that $N$ has nonnegative sectional curvature and positive $(2 m+2)$-th Ricci curvature. Now, we take a totally geodesic hypersurface $M=S^{2 m}(1) \times$ $R P^{2 m+1}(1)$ of $N$. Then $M$ divides $N$ into two open domains $\Omega_{1}$ and $\Omega_{2}$, where each has $M$ as its boundary. Theorem 2 tells us that any closed immersed minimal submanifold of dimension $\geqq 2 m+2$ in $\bar{\Omega}_{1}$ or $\bar{\Omega}_{2}$ is contained in $M$ and that there is no minimal embedded $4 m$-sphere in any of the closed domains $\bar{\Omega}_{1}$ and $\bar{\Omega}_{2}$.

When the ambient manifold is a Kaehler manifold, we have

THEOREM 3. Let $N^{2 n}(n \geqq 2)$ be a complete connected Kaehler manifold of nonnegative holomorphic bisectional curvature and of real dimension $2 n$. Let $W^{2 r}$ and $V^{t}$ be a complete immersed complex analytic submanifold of real dimension $2 r$ and $a t(\geqq 2 n-r)$-dimensional complete immersed totally geodesic submanifold, respectively, each immersed as a closed subset, and let one of $W^{2 r}$ and $V^{t}$ be compact. Assume $N^{2 n}$ has positive holomorphic bisectional curvature either at all points of $W^{2 r}$ or at all points of $V^{t}$. Then $W^{2 r}$ and $V^{t}$ must intersect.

Theorem 3 is also optimal in the sense that $V^{t}$ can't be weakened to be minimal, that the condition $r+t \geqq 2 n$ is essential and that the condition $N^{2 n}$ has positive holomorphic bisectional curvature on $W^{2 r}$ or $V^{t}$ is also necessary (see Examples 3, 4 and 5).

\section{Proofs of the results}

Proof of Theorem 1. We can assume that $V$ and $W$ are embedded, otherwise the proof given will then hold using any "sheet" of the immersion. Suppose then that $V$ and $W$ do not intersect. Let $\gamma:[0, l] \rightarrow N$ be a normal geodesic from $p \in W$ to $q \in V$ that realizes the minimum distance between these submanifolds (Since both $V$ and $W$ are closed subsets and one of them is compact, such $\gamma$ exists at least one). An argument using the first variation formula of arc-length shows that $\gamma$ strikes both $V$ and $W$ orthogonally. Since $V$ has codimension 1, parallel translating any unit vector $e$ tangent to $W$ at $p$ yields an unit vector field $E$ along $\gamma$ which is tangent to $V$ at $q$. This vector field along $\gamma$ gives rise to a "variation" of the geodesic $\gamma$ keeping end-points on the "endmanifolds" $V$ and $W$. Now, take a unit orthonormal basis $e_{1}, \cdots, e_{r}$ of $T_{p} W$. Parallel translating them along $\gamma$ give rise to $r$ mutually orthogonal unit vector fields $E_{1}, \cdots, E_{r}$ along $\gamma$. Each vector field $E_{\imath}(i=1, \cdots, r)$ makes a variation 
with length $L_{E_{i}}(s)$ of the variational curve of the geodesic $\gamma$ keeping endpoints on $V$ and $W$. The first variation of arc-length $L_{E_{i}}^{\prime}(0)$ is 0 . By the second variation formula of arc length [9, p. 99] we have, for $i=1, \cdots r$,

$$
L_{E_{i}}^{\prime \prime}(0)=\left\langle\sigma_{V}\left(E_{\imath}(q), E_{i}(q)\right), \gamma^{\prime}(l)\right\rangle-\left\langle\sigma_{W}\left(e_{\imath}, e_{\imath}, \gamma^{\prime}(0)\right\rangle-\int_{0}^{l} K\left(\gamma^{\prime}(t) \wedge E_{i}(t)\right) d t,\right.
$$

where $\sigma_{V}$ and $\sigma_{W}$ denote the second fundamental forms of $V$ and $W$, respectively. Since $V$ is totally geodesic, we have $\sigma_{V}=0$. From the minimallity of $W$, we know that

$$
\sum_{\imath=1}^{r} \sigma_{W}\left(e_{\imath}, e_{\imath}\right)=0 .
$$

Also, it follows from $\operatorname{Ric}_{(k)}(N) \geqq 0$ and $r \geqq k$ that

$$
\sum_{j=1}^{k} K\left(\gamma^{\prime}(t) \wedge E_{\imath_{j}}(t)\right) \geqq 0,
$$

for any indices $i_{1} \neq i_{2} \neq \cdots \neq i_{k} \in\{1, \cdots, r\}$, thus, we have

$$
\sum_{i=1}^{r} K\left(\gamma^{\prime}(t) \wedge E_{i}(t)\right)=\frac{r}{k C_{r}^{k}} \sum_{1 \leqq i_{1}<\cdots<i_{k} \leqq r} \sum_{j=1}^{k} K\left(\gamma^{\prime}(t) \wedge E_{\imath_{j}}(t)\right) \geqq 0 .
$$

Moreover, since $N$ has positive $k$-th Ricci curvature either at all points of $V$ or at all points of $W$, we know either

$$
\sum_{j=1}^{k} K\left(\gamma^{\prime}(0) \wedge E_{\imath j}(0)\right)>0,
$$

or

$$
\sum_{j=1}^{k} K\left(\gamma^{\prime}(l) \wedge E_{\imath_{j}}(l)\right)>0
$$

for any indices $i_{1} \neq \cdots \neq i_{k} \in\{1, \cdots, r\}$. Hence, we have either

$$
\sum_{i=1}^{r} K\left(\gamma^{\prime}(0) \wedge E_{i}(0)\right)>0
$$

or

$$
\sum_{i=1}^{r} K\left(\gamma^{\prime}(l) \wedge E_{i}(l)\right)>0 .
$$

Substituting these formulas into (1), we get

$$
\sum_{i=1}^{r} L_{E_{i}}^{\prime \prime}(0)=-\int_{0}^{\imath} \sum_{i=1}^{r} K\left(\gamma^{\prime}(t) \wedge E_{i}(t)\right) d t<0 .
$$

Thus, $L_{E_{i}}^{\prime \prime}(0)<0$, for some $i$, which contradicts to the assumption that $\gamma$ is of minimal length from $W$ to $V$. Hence $V$ and $W$ must intersect. This completes the proof of Theorem 1. Q.E.D. 
In 1951, S. B. Myers [10] proved that there exists no closed immersed minimal hypersurface in an open hemisphere. The following Corollary 1 which generalizes this result is a direct consequence of Theorem 1.

COROllary 1. Any immersed closed minimal submanifold in a Riemannian manifold $N$ of positive sectional curvature must intersect every immersed closed totally geodesic hypersurface of $N$.

Proof of Theorem 2. Since the Ricci curvature of $N^{n}$ is nonnegative and is positive at all points of $M^{n-1}$, we know that along each geodesic $\gamma:[0,+\infty)$ $\rightarrow N^{n}$ issuing orthogonally from $M^{n-1}$, the condition

$$
\int_{0}^{+\infty} \operatorname{Ric}\left(\gamma^{\prime}(t)\right) d t>0
$$

holds. Here, $\operatorname{Ric}\left(\gamma^{\prime}(t)\right)$ denotes the Ricci curvature of $N^{n}$ in the direction $\gamma^{\prime}(t)$. It then follows from Theorem 1 in [6] and the fact that $M^{n-1}$ is totally geodesic that $N^{n}$ is compact. We can thus conclude that the first Betti number of $N^{n}$ is zero by using again the fact that the Ricci curvature of $N^{n}$ is nonnegative and positive at some points. Combining this with the fact both $N^{n}$ and $M^{n-1}$ are orientable, and chasing through the exact sequences of homology groups, it is easy to see that $M^{n-1}$ divides $N^{n}$ into two components $\Omega_{1}$ and $\Omega_{2}$, such that $\partial \Omega_{1}=\partial \Omega_{2}$ (cf. [2]). This shows that the condition " $V^{r}$ is on one side of $M^{n-1}$ " makes sense. By Theorem 1, $V^{r}$ has the non-empty intersections of $M^{n-1}$. Thus, $V^{r}$ is on one side of $M^{n-1}$ and touches it. It then follows from Corollary 1.4 in [7] that $V^{r}$ is contained in $M^{n-1}$. When $r=n-2$, since $N^{n}$ has positive $k(k \leqq n-2)$-th Ricci curvature at all points of $M^{n-1}$ and $M^{n-1}$ is a totally geodesic hypersurface, we know that the Ricci curvature of $M^{n-1}$ is positive and that $V^{n-2}$ is a minimal hypersurface of $M^{n-1}$. By Frankel's theorem in [4], the natural homomorphism of the fundamental groups: $\pi_{1}\left(V^{n-2}\right) \rightarrow$ $\pi_{1}\left(M^{n-1}\right)$ is surjective. This completes the proof of Theorem 2 .

Proof of Theorem 3. Suppose that $W^{2 r}$ and $V^{t}$ do not intersect. Let $\gamma:[0, l] \rightarrow N^{2 n}$ be a normal geodesic from $p_{0} \in W^{2 r}$ to $q_{0} \in V^{t}$ that realizes the minimum distance between them. Denote by $J$ the complex structure of $N^{2 n}$. Since $W^{2 r}$ is a Kaehler submanifold, we can choose a unit orthonormal basis $e_{1}, \cdots, e_{r}, e_{r+1}, \cdots, e_{2 r}$ of $T_{p_{0}} W^{2 r}$ such that $e_{r+2}=J e_{\imath}(i=1, \cdots, r)$. Parallel translating $e_{1}, J e_{1}, \cdots, e_{r}$ and $J e_{r}$ along $\gamma$ give rise to $2 r$ mutually orthogonal unit vector fields $E_{1}, J E_{1}, \cdots, E_{r}$ and $J E_{r}$ along the geodesic. From $r+t \geqq 2 n$, we know that at least $r+1$ of the vector fields $E_{1}, J E_{1}, \cdots, E_{r}$ and $J E_{r}$ are tangent to $V^{t}$ at $q_{0}$. Thus, there exists some $k \in\{1, \cdots, r\}$ such that both $E_{k}$ and $J E_{k}$ are tangent to $V^{t}$ at $q_{0}$. The $E_{k}$ and $J E_{k}$ give rise to two variations of the geodesic $\gamma$ keeping endpoints on $W^{2 r}$ and $V^{t}$. The first variation of arclength $L_{E_{k}}^{\prime}(0)=L_{J E_{k}}^{\prime}(0)=0$, and using the second variation formula of arc-length, we get 


$$
L_{E_{k}}^{\prime \prime}(0)=\left\langle\sigma_{V t}\left(E_{k}\left(q_{0}\right), E_{k}\left(q_{0}\right)\right), \gamma^{\prime}(l)\right\rangle-\left\langle\sigma_{W 2 r}\left(e_{k}, e_{k}\right), \gamma^{\prime}(0)\right\rangle-\int_{0}^{l} K\left(E_{k} \wedge \gamma^{\prime}\right) d t,
$$

and

$$
\begin{aligned}
L_{J E_{k}}^{\prime \prime}(0)= & \left\langle\sigma_{V t}\left(J E_{k}\left(q_{0}\right), J E_{k}\left(q_{0}\right)\right), \gamma^{\prime}(l)\right\rangle-\left\langle\sigma_{W 2} r\left(J e_{k}, J e_{k}\right), \gamma^{\prime}(0)\right\rangle \\
& -\int_{0}^{l} K\left(J E_{k} \wedge \gamma^{\prime}\right) d t
\end{aligned}
$$

where, $\sigma_{W 2 r}$ and $\sigma_{V t}$ denote the second fundamental forms of $W^{2 r}$ and $V^{t}$, respectively. Since $W^{2 r}$ is a Kaehler submanifold and $V^{t}$ is totally geodesic, we have

$$
\sigma_{W 2 r}\left(J e_{k}, J e_{k}\right)=-\sigma_{W 2 r}\left(e_{k}, e_{k}\right), \quad \sigma_{V t}=0 .
$$

Hence, we have

$$
L_{E_{k}}^{\prime \prime}(0)+L_{J E_{k}}^{\prime \prime}(0)=-\int_{0}^{l} K\left(E_{k}(t) \wedge \gamma^{\prime}(t)\right) d t-\int_{0}^{l} K\left(J E_{k}(t) \wedge \gamma^{\prime}(t)\right) d t .
$$

Now, we denote by $R$ the Riemannian curvature tensor of $N^{2 n}$. For any point $p \in N^{2 n}$, recall that the holomorphic bisectional curvature $H\left(\sigma, \sigma^{\prime}\right)$ of two $J$ invariant planes $\sigma$ and $\sigma^{\prime}$ in $T_{p} N^{2 n}$ is defined by (cf. [8])

$$
H\left(\sigma, \sigma^{\prime}\right)=R(X, J X, Y, J Y),
$$

where $X$ is a unit vector in $\sigma$ and $Y$ a unit vector in $\sigma^{\prime}$. By Bianchi's identity we have

$$
K\left(E_{k}(t) \wedge \gamma^{\prime}(t)\right)+K\left(J E_{k}(t) \wedge \gamma^{\prime}(t)\right)=R\left(\gamma^{\prime}(t), J \gamma^{\prime}(t), E_{k}(t), J E_{k}(t)\right) .
$$

From the assumption that $N^{2 n}$ has nonnegative holomorphic bisectional curvature and has positive holomorphic bisectional curvature either at all points of $W^{2 r}$ or at all points of $V^{t}$, we have

$$
R\left(\gamma^{\prime}(t), J \gamma^{\prime}(t), E_{k}(t), J E_{k}(t)\right) \geqq 0,
$$

for any $t \in[0, l]$, and

$$
\max \left\{R\left(\gamma^{\prime}(0), J \gamma^{\prime}(0), E_{k}(0), J E_{k}(0)\right), R\left(\gamma^{\prime}(l), J \gamma^{\prime}(l), E_{k}(l), J E_{k}(l)\right)\right\}>0 .
$$

Substituting these formulas into (2), we get

$$
L_{E_{k}}^{\prime \prime}(0)+L_{J E_{k}}^{\prime \prime}(0)<0 \text {. }
$$

Hence, the second variation corresponding to at least one of the vector fields $E_{k}$ or $J E_{k}$ is strictly negative, contradicting to the assumption that $\gamma$ is of minimal length from $W^{2 r}$ to $V^{t}$. Thus, $W^{2 r}$ and $V^{t}$ must intersect. This completes the proof of Theorem 3. Q.E.D. 


\section{Examples}

In this section, we always assume $n \geqq 2$.

1. Let $S^{n+1}(1)=\left\{\left(x_{1}, \cdots, x_{n+2}\right) \in R^{n+2} \mid \sum_{\imath=1}^{n+2} x_{\imath}^{2}=1\right\}$ be the unit $(n+1)$-sphere. Take an $(n-1)$-dimensional totally geodesic submanifold $P=\left\{\left(0,0, x_{3}, \cdots, x_{n+2}\right)\right.$ $\left.\in S^{n+1}(1)\right\}$ and a Clifford minimal hypersurface $Q=\left\{\left(x_{1}, x_{2}, \cdots, x_{n+2}\right) \in S^{n+1}(1) \mid\right.$ $\left.x_{1}^{2}+x_{2}^{2}=1 / n, \sum_{\imath=3}^{n+2} x_{2}^{2}=(n-1) / n\right\}$ of $S^{n+1}(1)$. It is easy to see that $P \cap Q=\emptyset$. This example shows that the condition " $V$ is a totally geodesic hypersurface" in Theorem 1 can't be weakened to any of the two ones: (i) $V$ is a totally geodesic submanifold; (ii) $V$ is a minimal hypersurface.

2. The Riemannian product space $N=S^{2}(1) \times S^{2}(1)$ has nonnegative 2-th Ricci curvature. Take a totally geodesic hypersurface $M=S^{2}(1) \times S^{1}(1)$ and a minimal (in fact totally geodesic) surface $V=S^{2}(1) \times(0,0,1)$. Here, $S^{1}(1)=$ $\left\{\left(x_{1}, x_{2}, 0\right) \in S^{2}(1)\right\}$. It is easy to see that the 2-th Ricci curvature of $N$ can take value zero in some subspaces of the tangent spaces of $N$ at points of $M$ and $V$, but $M \cap V=\emptyset$. This example shows that if $N$ is only assumed to have nonnegative $k$-th Ricci curvature in Theorem 1 , then the conclusion is not valid.

3. Denote by $C P^{n}(c)$ the complex projective space with the Fubini-Study metric of holomorphic sectional curvature $c$. Let $\pi: S^{2 n+1}(1) \rightarrow C P^{n}(4)$ be the Hopf fibration. Let $\widetilde{W}=\left\{\left(z_{1}, z_{2}, 0, \cdots, 0\right) \in S^{2 n+1}(1) \subset C^{n+1} \mid z_{1} \bar{z}_{1}+z_{2} \bar{z}_{2}=1\right\}$ be a 3dimensional totally geodesic submanifold of $S^{2 n+1}(1)$ and $\tilde{V}=\left\{\left(z_{1}, z_{2}, \cdots, z_{n+1}\right) \in\right.$ $\left.\left.S^{2 n+1}(1)|| z_{1}\right|^{2}+\left|z_{2}\right|^{2}=3 / 2 n, \quad \sum_{l=3}^{n+1}\left|z_{2}\right|^{2}=(2 n-3) / 2 n\right\}$ be a Clifford minimal hypersurface of $S^{2 n+1}(1)$. Here, $\left(z_{1}, \cdots, z_{n+1}\right) \in C^{n+1}$ and we identify $C^{n+1}$ with $R^{2 n+2}$. Then $W=\pi(\widetilde{W})$ is a Kaehler submanifold of real dimension 2 and $V=\pi(\tilde{V})$ is a closed minimal hypersurface of $C P^{n}(4)$. Obviously, $\operatorname{dim}_{C} W+\operatorname{dim} V=2 n$, but $W \cap V=\emptyset$. This example shows that the condition " $V$ is totally geodesic" in Theorem 3 can't be weakened to "minimal".

4. Let $R P^{n}(c / 4) \rightarrow C P^{n}(c)$ be the natural totally geodesic imbedding of real projective space into the complex projective space: Using the homogeneous coordinate system $\left\{z_{1}, \cdots, z_{n+1}\right\}$ of $C P^{n}(c), R P^{n}(c / 4)$ is expressed by

$$
R P^{n}\left(\frac{c}{4}\right)=\left\{\left(z_{1}, \cdots, z_{n+1}\right) \in C P^{n}(c) \mid \bar{z}_{a}=z_{a}, a=1, \cdots, n+1\right\} .
$$

It is the fixed point set of the complex conjugation of the coordinates which is an isometry of $C P^{n}(c)$. Therefore it is totally geodesic. Now we take the standard complex quadric $Q^{n-1}(c) \rightarrow C P^{n}(c)$ :

$$
Q^{n-1}(c)=\left\{\left(z_{1}, \cdots, z_{n+1}\right) \in C P^{n}(c) \mid \sum_{i=1}^{n+1} z_{\imath}^{2}=0\right\} .
$$

It is easy to see that $\operatorname{dim} R P^{n}(c / 4)+\operatorname{dim}_{C} Q^{n-1}(c)=n+n-1=2 n-1<2 n$ and $R P^{n}(c / 4) \cap Q^{n-1}(c)=\emptyset$. This example shows that the condition on the dimensions of $V$ and $W$ in Theorem 3 is essential.

5. With the notations in the last example, let us consider $N=C P^{n}(c) \times$ $C P^{n}(c)$ with the product metric. Then $N$ has nonnegative holomorphic bisec- 
tional curvature. The complex analytic submanifold $W=C P^{n}(c) \times Q^{n-1}(c)$ and the totally geodesic submanifold $V=C P^{n}(c) \times R P^{n}(c)$ clearly satisfy the condition $\operatorname{dim}_{C} W+\operatorname{dim}_{R} V \geqq \operatorname{dim}_{R} N$, but $W \cap V=\emptyset$. This example shows the importance of the following condition in Theorem 3: $N$ has positive holomorphic bisectional curvature at all points of $V$ or $W$.

\title{
REFERENCES
}

[1] M. BERGER, Les varietes riemannienes homogenes normal simplement connexes a courbure strictement positive, Ann. Scuola Norm. Sup. Pisa, 15 (1961), 179246.

[2] H.I. Chol ANd A.N. WANG, A first eigenvalue estimate for minimal hypersurfaces, J. Differential Geom., 18 (1983), 559-562.

[3] T. Frankel, Manifolds with positive curvature, Pacific J. Math., 11 (1961), 165-171.

[4] T. Frankel, On the fundamental group of a compact minimal submanifold, Ann. of Math., 83 (1966), 68-73.

[5] J. Hadamard, Sur certaines propretes des trajectories en Dynamique, J. Math. Pures Appl. Serie 5 (1897), 331-387.

[6] G. Galloway, Some results on the occurances of compact minimal submanifolds, Manuscripta Math., 25 (1981), 209-219.

[7] G. Galloway and L. Rodriguez, Intersections of minimal submanifolds, Geom. Dedicata, 39 (1991), 29-42.

[8] S. I. Goldberg and S. Kobayashi, Holomorphic bisectional curvature, J. Differential Geom., 1 (1966), 225-233.

[9] W. Klingenberg, Riemannian Geometry, Berlin, New York, de Gruyter, 1982.

[10] S.B. Myers, Curvature of closed hypersurfaces and non-existence of closed minimal hypersurface, Trans. Amer. Math. Soc., 71 (1951), 211-217.

[11] J.L. Synge, The first and second variations of the length-integral in Riemannian space, Proc. London Math. Soc., 25 (1926), 247-264.

[12] H. Wu, Manifolds of partially positive curvature, Indiana Univ. Math. J., 36 (1987), 525-548.

\author{
Mathematical Institute \\ TOHOKu University, SENDAi 980-77 \\ JAPAN \\ Department of Mathematics \\ USTC, P.R. CHINA \\ AND \\ Mathematical Institute \\ Tohoku University, Sendai 980-77 \\ JAPAN
}

